\title{
Methods for studying transmembrane peptides in bicelles: consequences of hydrophobic mismatch and peptide sequence
}

\author{
Jennifer A. Whiles, Kerney J. Glover, Regitze R. Vold, ${ }^{1}$ and Elizabeth A. Komives* \\ University of California, San Diego 9500 Gilman Dr. La Jolla, CA 92093-0359, USA
}

\begin{abstract}
We have shown that bicelles prepared from dilauryl phosphatidylcholine (DLPC) and dipalmitoyl phosphatidylcholine (DPPC) align in a magnetic field under conditions similar to the more common dimyristoyl phosphatidylcholine (DMPC) bicelles. In addition, a model transmembrane peptide, P16, with a hydrophobic stretch of $24 \AA$, and specific alanine- $\mathrm{d}_{3}$ labels, was incorporated into all of the different bicelles. The long-chain phospholipid (DLPC, DMPC, or DPPC) remained unperturbed upon incorporation of the peptide while the quadrupolar splitting of the short-chain phospholipid along the bicelle rim increased by varying degrees in the different bicelle systems. The change in quadrupolar splitting of the short-chain phospholipids was attributed to changes in either fluidity of the planar region of the bicelle or differences in overall lipid packing. When the hydrophobic stretch of the bilayer was 22.8 (DMPC) or $26.3 \AA$ (DPPC), the peptide tilt was found to be transmembrane $\left(33-35^{\circ}\right.$ with respect to the bicelle normal). When the hydrophobic stretch of the bilayer was $19.5 \AA$ (DLPC), the peptide quadrupolar splittings suggested a loss of transmembrane orientation. When tryptophan was incorporated in the middle of the transmembrane region, the transmembrane orientation was also lost.
\end{abstract}

\section{Introduction}

Methods for understanding interactions between proteins and lipids are essential for elucidating biological structure-function relationships. Peptide-lipid interactions can affect both protein and bilayer structure. Previous studies have suggested that these interactions can serve several regulatory roles such as controlling the membrane-association of lytic peptides [1], modulating membrane-protein activity [2], promoting peptide aggregation [3-7], driving the formation of lipid microdomains, inducing segregation of proteins within the membrane [8-10], determining protein sorting within the secretion pathway [11,12], and allowing for cell membrane recognition or fusion [13-15].

\footnotetext{
${ }^{*}$ Corresponding author.

E-mail address: ekomives@ucsd.edu (E.A. Komives).

${ }^{1}$ Deceased.
}

Previous studies of model transmembrane peptides comprised of polyleucine or Ala-Leu repeats of varying lengths in model membrane systems have shown they form stable $\alpha$-helices [11,16-24]. These peptides commonly contain flanking tryptophans or lysines which are thought to act as anchoring residues that stabilize the helix within the bilayer and discourage peptide aggregation $[18,19,22,25]$. The $\mathrm{N}$ - and $\mathrm{C}$-termini are capped (acetylated and amidated, respectively) in order to stabilize the helix dipole and to mimic continuation of the membrane-spanning segment to other regions of a membrane incorporated protein.

Solid-state NMR and ATR-FTIR techniques have deduced peptide tilt angles of $10^{\circ}$ [26] and $0-35^{\circ}$ [23], respectively, for peptides in lipid bilayers of matching lengths. It also appeared that a hydrophobic mismatch of up to $10 \AA$ could be accommodated before radical deviations in transmembrane orientations or bilayered lipid structures occurred [11]. However, peptide orientations within a bilayer are thought to depend on a 
variety of sequence-specific factors such as overall hydrophobicity of the membrane-spanning region of the peptide, the length of the hydrophobic region, and the nature of the flanking residues.

Bicelles, phospholipid aggregates composed of a long-chain phospholipid bilayer surrounded by a rim of short-chain phospholipid, provide an opportunity to examine both peptide tilt and lipid packing as a function of hydrophobic thickness. Bicelles composed of dimyristoyl phosphatidylcholine (DMPC), under specific sample conditions, have been shown to spontaneously align with their normals perpendicular to the magnetic field [27-30]. In 1995, Sanders and Landis incorporated a lysine-flanked polyleucine peptide into DMPC/ DHexPC (dimyristoyl phosphatidylcholine/dihexanoyl phosphatidylcholine) and DMPC/CHAPSO ((cholamidopropyl)dimethylammonio-2-hydroxy-1-propane-sulfonate) bicelles [29]. This particular peptide, referred to as P16, contains a 16-residue hydrophobic sequence, resulting in a $24 \AA$ hydrophobic stretch. This length is compatible with that of a DMPC bilayer [31]. Chemical shift anisotropy measurements (CSA) for a ${ }^{13} \mathrm{C}$ labeled carbonyl in leucine were close to that predicted for a transmembrane helix parallel to the bicelle normal. However, neither the precise tilt angle nor the effects of the peptide on bicelle viability were examined.

In the work presented here, we prepared stable solutions of bicelles composed of various long-chain phospholipids in order to methodically examine peptide tilt and lipid packing. Solid-state ${ }^{2} \mathrm{H}$ NMR experiments showed that bicelles composed of dilauryl phosphatidycholine (DLPC) and dipalmitoyl phosphatidylcholine (DPPC) aligned in a magnetic field under conditions similar to their DMPC counterparts. Deuterium labeled P16 was successfully incorporated into DLPC, DMPC, and DPPC bicelles. In the case of a hydrophobic match between the peptide and the bilayer (DMPC bicelles), P16 was found to rest at a $33-35^{\circ}$ angle with respect to the bicelle normal (transmembrane). When tryptophan was incorporated in the middle of the transmembrane region, the peptide rested at a $71^{\circ}$ or $83^{\circ}$ angle with respect to the bicelle normal (nontransmembrane). A systematic study of changes both in the peptide tilt and in the lipid order upon hydrophobic mismatch is also presented.

\section{Materials and methods}

Materials. Fluorenyl-methoxycarbonyl (FMOC) amino acids and [O-(7-azabenzotriazol-1-yl)-1,1,3,3tetramethyluronium hexafluorophosphate] (HATU) were purchased from PE Biosystems (Foster City, CA). Deuterium depleted water and $\mathrm{L}$-alanine- $\left(3,3,3-\mathrm{d}_{3}, 99 \%\right)$ were obtained from Cambridge Isotope Labs (Andover, MA). $\mathrm{TmCl}_{3}$ was from Aldrich Chemicals. All other reagents were obtained from Fisher Scientific (Pittsburgh, PA), Aldrich Chemical (Milwaukee, WI), or VWR Scientific (Los Angeles, CA) and were used as received. DLPC, DMPC, DPPC, DHexPC, diheptanoyl phosphatidylcholine (DHepPC), and their fatty-acyl chain perdeuterated counterparts were obtained from Avanti Polar Lipids (Alabaster, AL) and used without further purification.

Peptide preparation. P16 (Ac-KKGLLLALLLLALL LALLLKKA- $\mathbf{N H}_{2}$ ) was synthesized using FMOC chemistry on a MilliGen 9050 solid-state peptide synthesizer using methods described previously [32]. Deuterium labels were introduced separately at positions A7, A12, and A16. Similar mutant peptides, L11W, were also prepared containing the same deuterium labels. The peptides were purified by reverse phase high performance liquid chromatography using a Phenomenex (Torrance, CA) Jupiter $\mathrm{C} 4$ column and a linear gradient at $1.5 \% / \mathrm{min}$ from $80: 20 \mathrm{H}_{2} \mathrm{O}$ :acetic acid to 80:20 1-butanol:acetic acid. The purity of the peptide was verified by MALDI-TOF mass spectrometry.

Sample preparation. Samples for solid-state NMR experiments were prepared by first dissolving the longchain phospholipid with or without peptide in $1.5 \mathrm{~mL}$ trifluoroethanol (TFE) to achieve an $R$ (peptide:longchain phospholipid) $=1: 50$. The TFE solution was then quickly injected into $10 \mathrm{~mL}$ of water at $50^{\circ} \mathrm{C}$. Samples were lyophilized for 2 days and then resuspended in a solution containing the appropriate amounts of shortchain phospholpid, ${ }^{2} \mathrm{H}$ depleted water, and $1.0 \mathrm{M} 2-[\mathrm{N}-$ morpholino]ethanesulfonic acid (Mes) buffer (pH 6.0) to achieve the desired ratio of long:short-chain phospholipid ( $q=3.3$ for DLPC bicelles, $q=3.5$ for DMPC bicelles, and $q=3.0$ for DPPC bicelles), total phopholipid concentration $\left(c_{\mathrm{L}}\right)$ of $20 \%(\mathrm{w} / \mathrm{w})$, and a final buffer concentration of $25 \mathrm{mM}$. The samples were vortexed and briefly centrifuged (low speed, room temperature). Repeating this cycle 2-3 times, as well as heating the sample to $50^{\circ} \mathrm{C}$ and rapid cooling in liquid $\mathrm{N}_{2}$, facilitated homogeneous mixing. The sample to which lanthanides were added contained a 1:15 molar ratio of the chelating lipid, 1,2,-dimyristoyl-sn-glycero-3-phosphoethanolaminediethylenetriaminepentaacetate (DTPA， $1096.35 \mathrm{~g} /$ mol, Avanti Polar Lipids):DMPC and a 1:1 molar ratio of $\mathrm{Tm}^{3+} \mathrm{Cl}_{3}$ :DTPA. When added together, the $\mathrm{Tm}^{3+}$ is chelated by the DTPA and this results in the same effect on the magnetic succeptibility of the lipids without interaction of the $\mathrm{Tm}^{3+}$ with peptides in the bicelle [33]. Preliminary experiments were performed to determine the minimum concentration of $\mathrm{Tm}^{3+}$ added to bicelles containing DTPA to cause them to "flip" and this concentration was used in all experiments.

Circular dichroism. Circular dichroism (CD) spectra were obtained at $37^{\circ} \mathrm{C}$ or $45^{\circ} \mathrm{C}$ (depending upon the $T_{\mathrm{m}}$ of the phospholipid) on an Aviv Circular Dichroism Spectrometer Model 202. The samples were 4\% (w/w) 
total lipid (0.5 DMPC:DHPC molar ratio). Five mM Mes buffer, $\mathrm{pH}$ 6.0, and a 1:100 peptide:DMPC ratio. For other long-chain lipids, the same ratios were used.

Solid-state ${ }^{2} H$ NMR. Deuterium quadrupole echo spectra [34] were acquired at $55.3 \mathrm{MHz}$ on a Chemagnetics CMX-250/360 spectrometer interfaced to an ENI LPI-10 rf amplifier and an 8.5 T Oxford Instruments magnet. The spectrometer was controlled by a Sun SPARCstation 5 equipped with the Spinsight 3.0 software package (Chemagnetics, Fort Collins, CO). The sample temperature was maintained with a LakeShore $91 \mathrm{C}$ controller. A standard quadrupole echo sequence, $\pi / 2-\tau-\pi / 2-\tau_{1}-a c q$, was used with an acquisition of 4096 data points when observing deuterated lipid and 16,384 data points when observing the labeled peptide. Experiments were averaged over enough transients to provide an adequate ratio of signal-to-noise. Parameters common in both types of experiments were $\tau=50.0 \mu \mathrm{s}$, $\tau_{1}=35.0 \mu \mathrm{s}$, a $\pi / 2$ pulse length of $2.1 \mu \mathrm{s}$, and a spectral width of $500 \mathrm{kHz}$ to facilitate location of the echo maximum. Data processing included fractional left shifting, zero filling, and multiplication by an exponential of the second half of the quadrupole echo prior to Fourier transformation. For consistency, all spectra involving $\mathrm{P} 16$ were acquired at $45^{\circ} \mathrm{C}$, at which point all bicelle types were well aligned. In other work, we have shown that for long-chain lipids with lower $T_{\mathrm{m}}$ 's such as DMPC, no differences are seen when spectra are collected at $37^{\circ} \mathrm{C}$ vs $45^{\circ} \mathrm{C}$ [35].

Determination of peptide tilt. As determined previously for bicelles [36], and as discussed thoroughly in Whiles et al. [37], the observed quadrupolar splitting for a deuterated alanine residue in a bicelle-associated helical peptide $\left(\triangle^{\mathrm{P}}\right)$ can be expressed as

$\pm \Delta^{\mathrm{P}}=(84 \mathrm{kHz}) * S_{\mathrm{pw}} S_{\mathrm{\alpha n}} S_{\mathrm{nN}} S_{\mathrm{Nl}}$,

where $S_{\mathrm{Nl}}, S_{\mathrm{pw}}, S_{\mathrm{\alpha n}}$, and $S_{\mathrm{nN}}$ are order parameters dependent upon the P16 peptide and the type of bicelle used in the study. As mentioned earlier, bicelles align with their normals perpendicular to the magnetic field, resulting in an order parameter, $S_{\mathrm{Nl}},=1 / 2 . S_{\mathrm{nN}}$ can be determined as shown in Eq. (2)

$S_{\mathrm{nN}}=\Delta_{\mathrm{BIC}}^{\mathrm{L}} / \Delta_{\mathrm{MLV}}^{\mathrm{L}}$,

where $\Delta_{\mathrm{BIC}}^{\mathrm{L}}$ and $\Delta_{\mathrm{MLV}}^{\mathrm{L}}$ are the plateau deuteron quadrupolar splittings for the long-chain phospholipid in a bicelle and multilamellar vesicle (MLV), respectively. $S_{\mathrm{pw}}$ is the molecular order parameter that describes any conformational instability or overall peptide wobble. Previous studies have shown that transmembrane domains have relatively high order parameters [38-40] and studies of membrane-associated peptides in bicelles have also revealed high order parameters [37]. Therefore, a value of 1 for $S_{\mathrm{pw}}$ was used here. $S_{\alpha \mathrm{n}}$ is the order parameter arising from uniaxial bicellar spinning about the membrane normal and, according to the framework outlined previously [37], is the order parameter from which we can determine our peptide tilt within the membrane. Assuming P16 forms a stable and rigid helix within the bicelle, the tilt angle of the peptide with respect to the bicelle normal $(t)$ and the fixed degree of rotation $(r)$ of the helix about its axis can be described by a total angle $\beta_{\alpha \mathrm{n}}$ using the following relations:

for $\mathrm{A} 7, \quad \cos \beta_{\alpha \mathrm{n}}=-\sin 56^{\circ} \cos (r) \sin t$

$$
+\cos 56^{\circ} \cos t
$$

for A12, $\quad \cos \beta_{\alpha \mathrm{n}}=-\sin 56^{\circ} \cos \left(r+220^{\circ}\right) \sin t$

$$
+\cos 56^{\circ} \cos t
$$

for A16, $\quad \cos \beta_{\alpha \mathrm{n}}=-\sin 56^{\circ} \cos \left(r+180^{\circ}\right) \sin t$

$$
+\cos 56^{\circ} \cos t \text {. }
$$

$\beta_{\alpha \mathrm{n}}$ can subsequently be related to the following experimentally determined order parameter:

$S_{\text {an }}=\left\langle 1 / 2\left(3 \cos ^{2} \beta_{\text {an }}-1\right)\right\rangle$.

An in-house $\mathrm{C}++$ computer program allowed computation of the tilt angles (varied in $1^{\circ}$ intervals from $0^{\circ}$ to $90^{\circ}$ ) and the rotation angles (varied in $1^{\circ}$ increments from $0^{\circ}$ to $360^{\circ}$ ) that were consistent with the quadrupolar splitting of all three alanine labels from

$\pm \Delta^{\mathrm{P}}=(84 \mathrm{kHz}) * S_{\mathrm{pw}}\left\langle 1 / 2\left(3 \cos ^{2} \beta_{\text {an }}-1\right)\right\rangle S_{\mathrm{nN}} S_{\mathrm{Nl}}$,

where $\cos \beta_{\alpha n}$ is derived from Eqs. (3a)-(3c).

The program then ranked all possible tilt and rotation angle combinations based on how far the individual and average quadrupolar splittings varied from the theoretical values for a particular tilt and rotation. Although the program gives both a tilt and a rotation angle, only the tilt angle is relevant.

\section{Results and discussion}

DLPC and DPPC bicelles. DLPC/DHexPC and DPPC/DHepPC bicelles were prepared with $q=3.3$ and 3.0 , respectively. The type of short-chain lipid required was determined experimentally based on its ability to solubilize the long-chain phospholipid. The maximum $q$ that resulted in complete solubilization of the long-chain lipid was used to maximize bicelle alignment in the magnetic field, which depends on the magnetic susceptibility of the long-chain phospholipid methylene chains. Solid-state ${ }^{2} \mathrm{H}$ NMR spectra of the chain perdeuterated long-chain phospholipid in DLPC and DPPC bicelles are shown in Figs. 1 and 2, respectively. The A panels in both figures demonstrate the temperature-dependent alignment of the bicelles. As in the case with DMPC bicelles, both DLPC and DPPC bicelles align at temperatures above the phase transition temperature $\left(T_{\mathrm{m}}\right)$ of the long-chain phospholipids (Table 1 ). In addition, the 

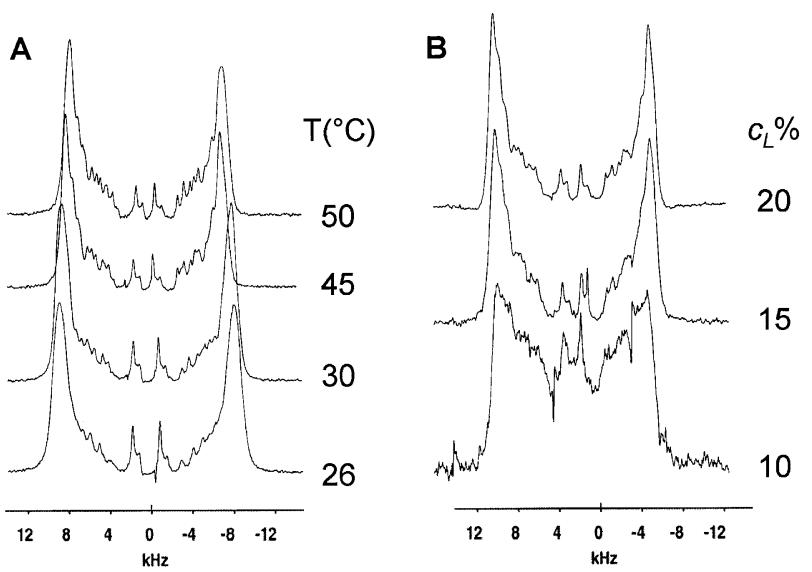

Fig. 1. Solid-state ${ }^{2} \mathrm{H}$ NMR spectra of DLPC- $\mathrm{d}_{46} / \mathrm{DHexPC}$ bicelles $(q=3.3)$ as a function of (A) temperature and (B) phospholipid concentration $\left(c_{\mathrm{L}}(\mathrm{w} / \mathrm{w} \%)\right)$. All samples contained $25 \mathrm{mM}$ Mes, $\mathrm{pH} 6.0$. Spectra were acquired at $45^{\circ} \mathrm{C}$.
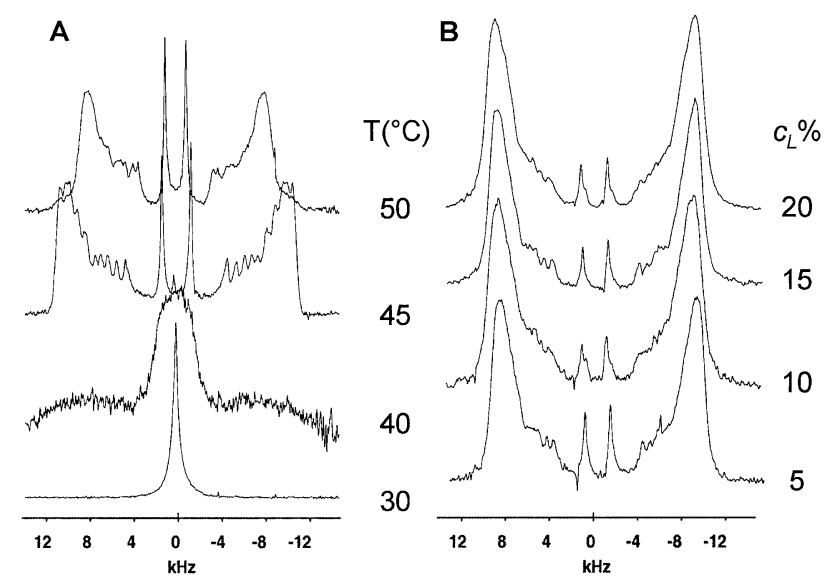

Fig. 2. Solid-state ${ }^{2} \mathrm{H}$ NMR spectra of DPPC- $\mathrm{d}_{62} / \mathrm{DHepPC}$ bicelles $(q=3.0)$ as a function of (A) temperature and (B) phospholipid concentration $\left(c_{\mathrm{L}}\left(\mathrm{w} / \mathrm{w}^{0} \%\right)\right)$. All samples contained $25 \mathrm{mM}$ Mes, $\mathrm{pH}$ 6.0. Spectra were acquired at $45^{\circ} \mathrm{C}$.

B panels in each figure reveal the concentration-dependent alignment, both of which follow trends previously observed for DMPC bicelles [41]. DPPC bicelles remain more aligned at lower phospholipid concentrations than DLPC bicelles, most likely due to their longer methylene chains.

DPPC bicelles were also prepared at $q=0.5$, a phase which does not align in the magnetic field (data not shown). These bicelles behaved similarly to their DMPC counterparts and have shown potential for use in studying enzymatic hydrolysis by phospholipase $\mathrm{A}_{2}$ [42]. It is predicted that DLPC bicelles will exhibit similar morphology and behavior.

Determination of bicelle wobble from ${ }^{2} \mathrm{H} N M R$. As shown in Eq. (2), $S_{\mathrm{nN}}$ for DLPC, DMPC, and DPPC bicelles was determined by taking the ratio of the quadrupolar splitting for the long-chain phospholipid in
Table 1

Composition and bilayer properties of various bicelle systems

\begin{tabular}{llll}
\hline Bicelle type & $q$ & $\begin{array}{l}\text { Hydrophobic } \\
\text { thickness }(\AA)\end{array}$ & $T_{\mathrm{m}}\left({ }^{\circ} \mathrm{C}\right)$ \\
\hline DLPC/DHexPC & 3.3 & $19.5^{\mathrm{a}}$ & $0^{\mathrm{c}}$ \\
DMPC/DHexPC & 3.5 & $22.8^{\mathrm{b}}$ & $24.0^{\mathrm{d}}$ \\
DPPC/DHepPC & 3.0 & $26.3^{\mathrm{b}}$ & $41.4^{\mathrm{d}}$ \\
\hline
\end{tabular}

${ }^{\mathrm{a}}$ See Ref. [47].

${ }^{\mathrm{b}}$ See Ref. [31].

${ }^{\mathrm{c}}$ See Ref. [48].

${ }^{\mathrm{d}}$ See Ref. [49].

a bicelle to the splitting at the $90^{\circ}$ edge of the powder pattern in MLVs (data not shown). These values were found to be 0.73 for DLPC bicelles, 0.84 for DMPC bicelles, and 0.86 for DPPC bicelles. This increase in $S_{\mathrm{nN}}$ as a function of chain length is reasonable considering that bicelle alignment in the magnetic field is due to the magnetic susceptibility of the long-chain phospholipid, which is additive with methylene chain length.

P16 tilt and effects on phospholipid order. Circular dichroism spectra acquired for P16 in DLPC/DHexPC, DMPC/DHexPC, and DPPC/DHepPC are shown in Fig. 3. The peptide exhibits a strong $\alpha$-helical signature in all three systems studied and the spectra indicate that there is no change in peptide conformation as a function of lipid type.

Fig. 4 shows solid-state deuterium NMR spectra for P16-A12d $\mathrm{d}_{3}$ in DLPC, DMPC, and DPPC bicelles (panels A, B, and C, respectively). From these data we can see that it is possible to obtain well-aligned peptide spectra in all three bicelle systems. The specific quadrupolar splittings for all three deuterium labels for P16

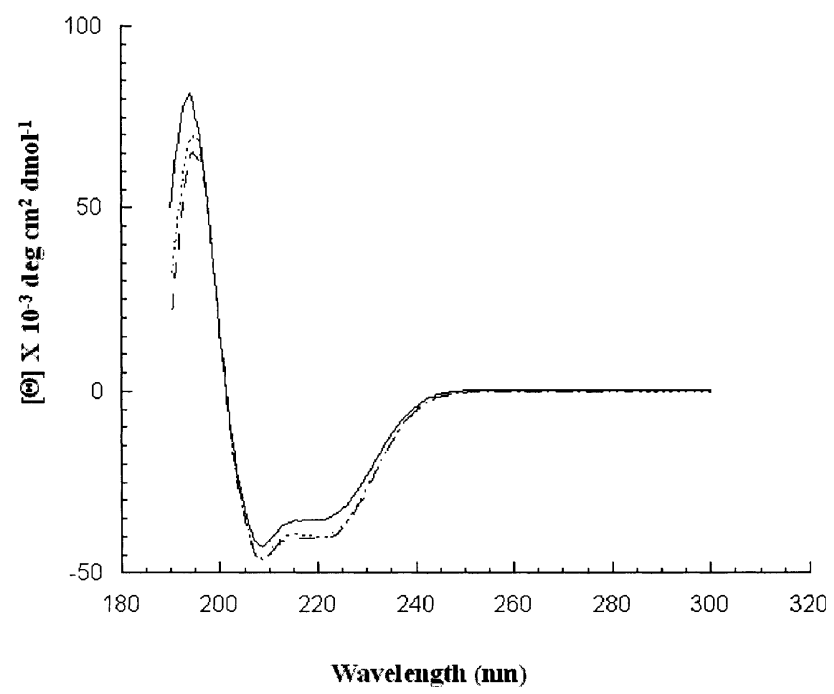

Fig. 3. CD spectra of P16 in DLPC (dotted line), DMPC (solid line), and DPPC (dashed line) bicelles. Samples contained 3\% (w/w) total lipid $(q=0.5), 25 \mathrm{mM}$ Hepes, $\mathrm{pH} 7.5$, and $R=1: 100$. DLPC and DMPC spectra were acquired at $37^{\circ} \mathrm{C}$ and the DPPC spectrum was acquired at $45^{\circ} \mathrm{C}$. 


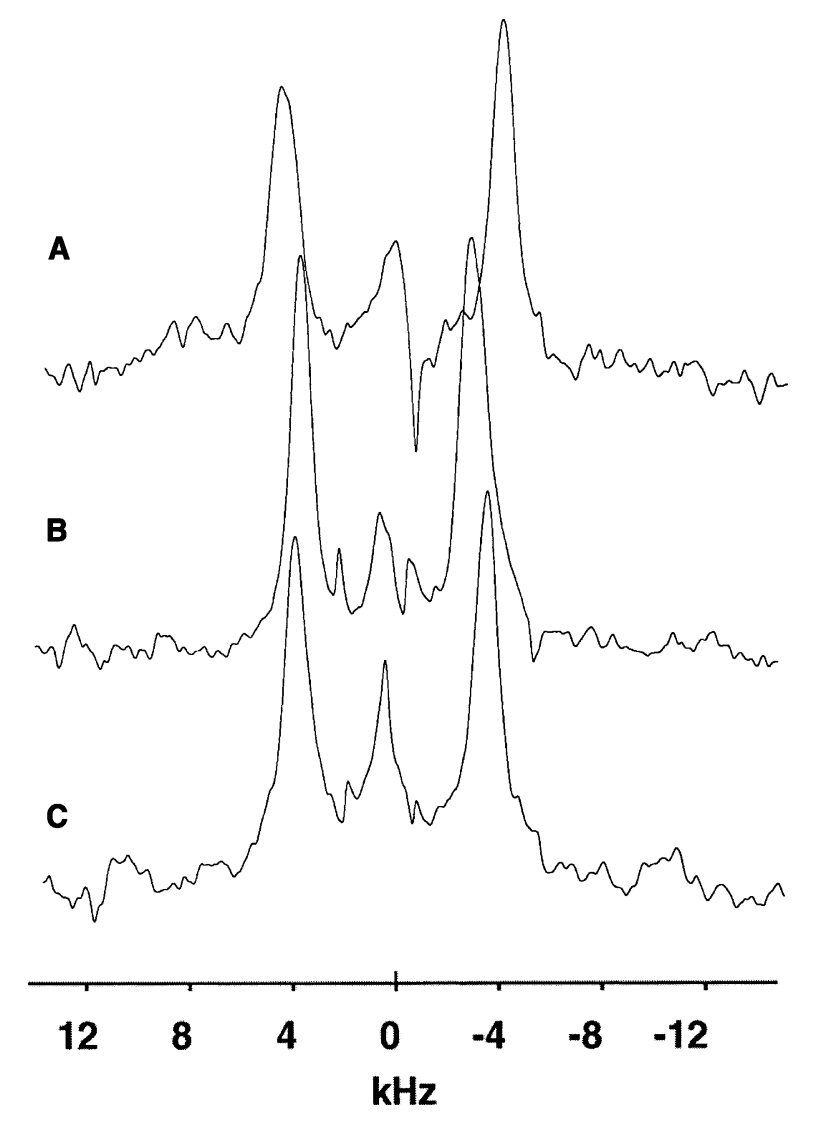

Fig. 4. Solid-state ${ }^{2} \mathrm{H}$ NMR spectra of $\mathrm{P} 16-\mathrm{A} 12 \mathrm{~d}_{3}$ in (A) DLPC/ DHexPC $(q=3.3)$, (B) DMPC/DHexPC $(q=3.5)$, and (C) DPPC/ DHepPC $(q=3.0)$ bicelles. All samples contained $20 \%(\mathrm{w} / \mathrm{w})$ total lipid, $25 \mathrm{mM}$ Hepes, $\mathrm{pH} 6.0$, and $R=1: 50$. Spectra were acquired at $45^{\circ} \mathrm{C}$.

in DMPC bicelles are shown in Table 2. When the quadrupolar splittings were entered into Eq. (5) using the $\mathrm{C}++$ program as described in the Section 2, P16 was found to rest at a $33-35^{\circ}$ angle with respect to the bicelle normal (DMPC bicelles). The quadrupolar splitting for $\mathrm{P} 16-\mathrm{A} 12 \mathrm{~d}_{3}$ in DPPC bicelles was similar to that in DMPC bicelles, suggesting that increasing the hydrophobic stretch of the bicelle compared to that of the peptide had no affect on the peptide orientation within the bicelle. However, the quadrupolar splitting of P16A12 $d_{3}$ in DLPC bicelles was greater than that observed in DMPC bicelles. This change in splitting could be due to the adoption of a slightly more nontransmembrane orientation (closer to parallel to the bicelle surface) by the peptide or differences in incorporation of the peptide within the bicelle.

Table 2

Peptide quadrupolar splittings in DMPC bicelles $(\mathrm{kHz})$ at $45^{\circ} \mathrm{C}$

\begin{tabular}{lrc}
\hline Label position & P16 & P16-L11W \\
\hline A7 & 1.8 & 7.6 \\
A12 & 7.0 & 12.7 \\
A16 & 10.3 & 13.6 \\
\hline
\end{tabular}

The quadrupolar splittings for $\mathrm{P} 16-\mathrm{L} 11 \mathrm{~W}$ are also shown in Table 2. Computation of the tilt angle from the quadrupolar splitting values for P16-L11W resulted in very different tilt angles as compared with P16. P16$\mathrm{L} 11 \mathrm{~W}$ was found to rest at a nontransmembrane orientation $\left(73^{\circ}\right.$ or $82^{\circ}$ tilt angle with respect to the bicelle normal) in DMPC bicelles. It is believed that in this latter case, the tryptophan residue is able to control the association of the peptide with the bicelle. This result is not surprising given previous studies that have shown clustering of aromatic residues at the memebrane-water interface [43-46]. However, this result does demonstrate the power of one amino acid in dominating lipid-peptide interactions as well as the need for careful consideration when constructing putative transmembrane domains.

Solid-state ${ }^{2} \mathrm{H}$ NMR also provides the opportunity to examine the affects of peptide incorporation on bicelle stability and lipid packing. Fig. 5 shows spectra of fatty acid perdeuterated long-chain phospholipid in bicelles in the presence and absence of P16. Except for DLPC, P16 appears to cause minor perturbations in the resolution of all three long-chain phospholipid spectra and slight increases in the plateau splittings. If we allow for deviations due to sample variability, these changes are not likely to be significant and indicate that bicelle viability is maintained.

In contrast, P16 induces significant changes in both the resolution and the quadrupolar splittings of all the deuterons in the fatty acid perdeuterated short-chain phospholipids of all bicelles (Fig. 6). The effect becomes more pronounced as the length of the long-chain phospholipids is increased. The plateau quadrupolar splittings of the rim lipids of DPPC bicelles increase more than previously predicted possible for phospholipids on the rim of a bicelle [30]. These results were also observed for P16-L11W. These spectra would at first seem to indicate that P16 is interacting directly with the shortchain phospholipids. However, several further experiments suggest that this is not the case. First, control experiments in which P16 was incorporated into DHexPC micelles (i.e., short-chain phospholipids only) did show lipid:peptide nuclear overhauser effects (NOEs) [35]. Second, NOEs between P16 and the longchain DMPC lipids were observed when P16 was reconstituted into unaligned bicelles $(q=0.5)$ containing combinations of fatty acid perdeuterated and nonlabeled phospholipids [35]. In the unaligned bicelles, NOEs were absent between P16 and the DHexPC. Since NOEs were not observed in the unaligned bicelles (that contain a 2:1 molar ratio of DHexPC:long chain) but were observed between P16 and DHexPC when that was the only lipid present, these results suggest that the majority of P16 is interacting with the long-chain lipid in the planar region of the bicelle. However, it is not possible a priori to rule out that the weakening of peptide-DHexPC NOEs may at least in part be caused 
by the rapid exchange of DHexPC on and off the bicelles and/or by the shorter lifetime of DHexPC-peptide interactions vs that of DMPC-peptide contacts.

To explore the affect of P16 on the DHexPC, we sought some way to confirm that the bicelle morphology remained intact when P16 was present. If the bicelles

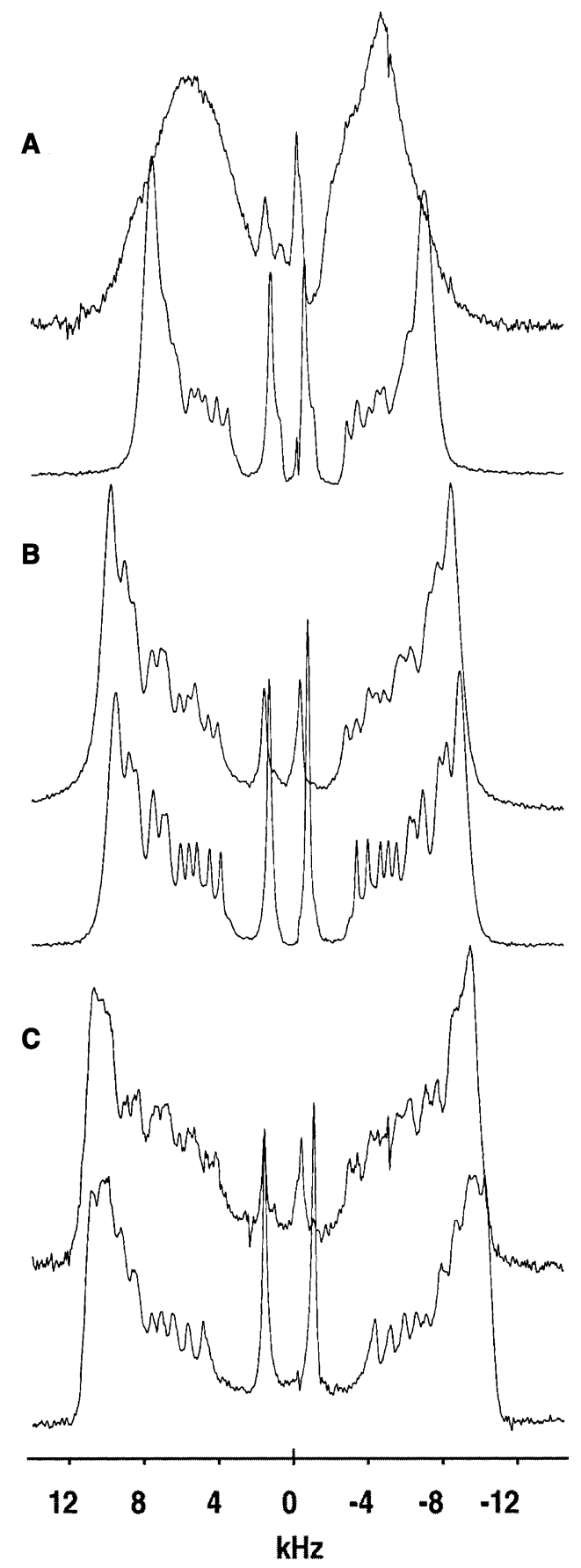

Fig. 5. Solid-state ${ }^{2} \mathrm{H}$ NMR spectra of fatty-acid perdeuterated longchain phospholipids in in DLPC bicelles (A), DMPC bicelles (B), or DPPC bicelles (C) in the presence (top) and absence (bottom) of P16. All samples contained $20 \%(\mathrm{w} / \mathrm{w})$ total lipid $(q=3.3$ for DLPC bicelles, $q=3.5$ for DMPC bicelles, and $q=3.0$ for DPPC bicelles), $R=1: 50$, and $25 \mathrm{mM}$ Mes, $\mathrm{pH}$ 6.0. Spectra were acquired at $45^{\circ} \mathrm{C}$. retained their disk-like shape and size, they should rotate rapidly around their normals and this can be confirmed by "flipping" them. Bicelles prepared with a chelating lipid, DTPA, chelated to $\mathrm{Tm}^{3+}$, a lanthanide agent, align with their normals parallel to the magnetic field because $S_{\mathrm{N} 1}$ is now 1 instead of $1 / 2$ (cf. Eq. (1)) [33].

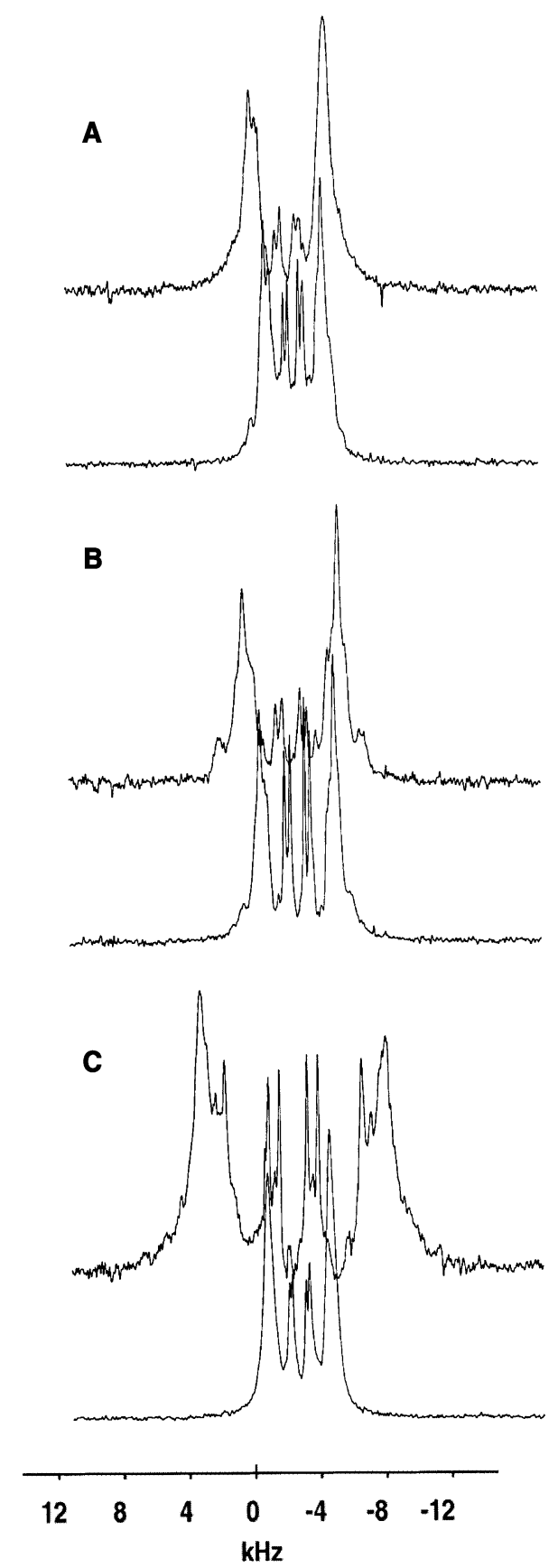

Fig. 6. Solid-state ${ }^{2} \mathrm{H}$ NMR spectra of fatty-acid perdeuterated shortchain phospholipids in bicelles in DLPC bicelles (A), DMPC bicelles (B), or DPPC bicelles (C) in the presence (top) and absence (bottom) of P16. All samples contained $20 \%(\mathrm{w} / \mathrm{w})$ total lipid $(q=3.3$ for DLPC bicelles, $q=3.5$ for DMPC bicelles, and $q=3.0$ for DPPC bicelles), $R=1: 50$, and $25 \mathrm{mM}$ Mes, $\mathrm{pH}$ 6.0. Spectra were acquired at $45^{\circ} \mathrm{C}$. 


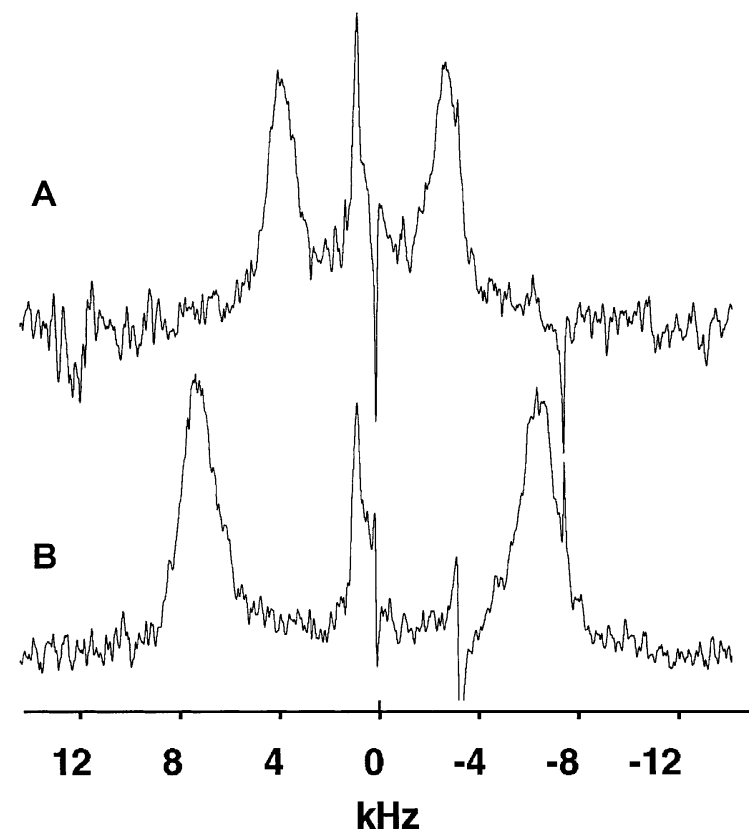

Fig. 7. Solid-state ${ }^{2} \mathrm{H}$ NMR spectra of P16-L11W-A7 $\mathrm{d}_{3}$ in DMPC/ DHexPC bicelles (A) or the same containing 1:15 DTPA:DMPC and to which a 1:1 molar ratio of $\mathrm{TmCl}_{3}$ :DTPA was added (B) $\left(q=3.5, c_{\mathrm{L}}=20 \%(\mathrm{w} / \mathrm{w})\right.$ total lipid). Samples contained $25 \mathrm{mM}$ Hepes, $\mathrm{pH} 6.0$, and $R=1: 50$. Spectra were acquired at $37^{\circ} \mathrm{C}$.

We were able to incorporate P16-L11W into DTPA: $\mathrm{Tm}^{3+}$ containing bicelles. The quadrupolar splitting of P16-L11W in DMPC bicelles doubles (Fig. 7), as would be expected if the bicelles are spinning rapidly around their normals. This experiment indicates that changes observed in the short-chain lipid spectra upon the addition of P16-L11W are not likely to be due to loss of bicelle morphology but are instead indirectly caused by changes in either lipid packing or bilayer fluidity. It is also interesting to note that both P16 and P16-L11W cause similar perturbation of the DHexPC, despite the fact that their orientations in the planar region are very different. This also lends credence to the idea that the changes in the DHexPC are a result of general ordering of the lipid rather than any specific interaction with the DHexPC.

\section{Conclusions}

In this work we have shown that bicelles of varying thickness can be prepared and utilized to study the structure and orientation of transmembrane domains. These bicelles are particularly useful in cases where the peptide hydrophobic length is longer than that of the bilayer. We have also shown how one amino acid, namely a tryptophan, can dictate the association of a membrane-associated peptide with a bilayer. Mismatches in bilayer thickness relative to the peptide hydrophobic length result in retention of helical structure but loss of transmembrane orientation. The observation that a single tryptophan or a hydrophobic mismatch can alter peptide orientation demonstrates the utility of solid-state deuterium NMR in determining the structural orientation of transmembrane domains. Also, the fact that both the peptide and the lipids can be studied under identical sample conditions provides important information about the interaction between the membrane and the transmembrane domain. Finally, bicelles provide a powerful tool for studying peptide orientation, phospholipid packing, and membrane-peptide interactions together in samples of similar composition.

\section{Acknowledgments}

This work was supported by an NIH (5 R01 GM54034) grant to RRV. JAW was supported by a La Jolla Interfaces in Science predoctoral fellowship through the Burroughs Wellcome Fund.

\section{References}

[1] N. Mobashery, C. Nielsen, O.S. Andersen, FEBS Lett. 412 (1997) $15-20$.

[2] A. Johannson, C.A. Keigthley, G.A. Smith, C.D. Richards, T.R. Hesketh, J.C. Metcalfe, J. Biol. Chem. 256 (1981) 1643-1650.

[3] J.A. Killian, Biochim. Biophys. Acta 1376 (1998) 401-416.

[4] J.C. Owicki, M.W. Springgate, H.M. McConnel, Proc. Natl. Acad. Sci. USA 75 (1978) 1616-1619.

[5] O.G. Mouritsen, M. Bloom, Biophys. J. 46 (1984) 141-153.

[6] D.R. Fattal, A. Ben-Shaul, Biophys. J. 65 (1993) 1795-1809.

[7] S. May, A. Ben-Shaul, Biophys. J. 76 (1999) 751-767.

[8] M.M. Sperotto, O.G. Mouritsen, Eur. Biophys. J. 22 (1993) 323-328.

[9] D. Marsh, Mol. Membr. Biol. 12 (1995) 59-64.

[10] J.Y.A. Lehtonen, P.K.J. Kinnunen, Biophys. J. 72 (1997) $1247-1257$.

[11] R.J. Webb, J.M. East, R.P. Sharma, A.G. Lee, Biochemistry 37 (1998) 673-679.

[12] H.R. Pelham, S. Munro, Cell 75 (1993) 603-605.

[13] D.P. Siegel, R.M. Epand, Biophys. J. 73 (1997) 3089-3111.

[14] B. Bechinger, Proteins: Struct. Funct. Gen. 27 (1997) 481-492.

[15] B. Ghebremariam, V. Sidorov, S. Matile, Tetrahedron Lett. 40 (1999) $1445-1448$.

[16] M. Bloom, E. Evans, O.G. Mouritsen, Quart. Rev. Biophys. 24 (1991) 293-297.

[17] J.H. Davis, Biochim. Biophys. Acta 737 (1983) 117-171.

[18] J.C. Huschilt, B.M. Millman, J.H. Davis, Biochim. Biophys. Acta 979 (1989) 139-141.

[19] J.A. Killian, I. Salemink, M.R.R. de Planque, G. Lindblom, R.E. Koeppe II, D. Greathouse, Biochemistry 35 (1996) 1037-1045.

[20] S.L. Jianhua Ren, Z. Wang, E. London, Biochemistry 36 (1997) 10213-10220.

[21] S.L. Jianhua Ren, J. Wang, E. London, Biochemistry 38 (1999) $5905-5912$

[22] P.C.A. van der Wel, T. Pott, S. Morein, D. Greathouse, R.E. Koeppe II, J.A. Killian, Biochemistry 39 (2000) 3124-3133.

[23] U. Harzer, B. Bechinger, Biochemistry 39 (2000) 13106-13114.

[24] S. Morein, R.E. Koeppe II, G. Lindblom, B. de Kruijff, J.A. Killian, Biophys. J. 78 (2000) 2475-2485. 
[25] F.A. Nezil, M. Bloom, Biophys. J. 61 (1992) 1176-1183.

[26] M.R.R. de Planque, E. Goormaghtigh, D. Greathouse, R.E. Koeppe II, J.A.W. Kruijtzer, R.M.J. Liskamp, B. de Kruijff, J.A. Killian, Biochemistry 40 (2001) 5000-5010.

[27] C.R. Sanders, B.J. Hare, K.P. Howard, J.H. Prestegard, Progr. NMR Spectr. 26 (1994) 421-444.

[28] C.R. Sanders, J.H. Prestegard, Biophys. J. 58 (1990) 447-460.

[29] C.R. Sanders, G.C. Landis, Biochemistry 43 (1995) 4030-4040.

[30] R.R. Vold, R.S. Prosser, J. Magn. Reson. B 113 (1996) 267-271.

[31] M.M. Sperotto, O.G. Mouritsen, Eur. Biophys. J. 16 (1988) 1-10.

[32] K.J. Glover, P.M. Martini, R.R. Vold, E.A. Komives, Anal. Biochem. 272 (1999) 270-274.

[33] R.S. Prosser, H. Bryant, R.G. Bryant, R.R. Vold, J. Magn. Reson. 141 (1999) 256-260.

[34] J.H. Davis, K.R. Jeffrey, M. Bloom, M.I. Valic, T.P. Higgs, Chem. Phys. Lett. 42 (1976) 390-394.

[35] K.J. Glover, J.A. Whiles, R.R. Vold, G. Melacini, J. Biomol. NMR 22 (2002) 57-64.

[36] S.R. Prosser, J.S. Hwang, R.R. Vold, Biophys. J. 74 (1998) 2405-2418.
[37] J.A. Whiles, R. Brasseur, K.J. Glover, G. Melacini, E.A. Komives, R.R. Vold, Biophys. J. 80 (2001) 280-293.

[38] R.E. Koeppe II, J.A. Killian, D.V. Greathouse, Biophys. J. 66 (1994) 14-24.

[39] R.S. Prosser, S.I. Daleman, J.H. Davis, Biophys. J. 66 (1994) $1415-1428$.

[40] D.H. Jones, K.R. Barber, E.W. VanDerLoo, C.W.M. Grant, Biochemistry 37 (1998) 16780-16787.

[41] J.O. Struppe, R.R. Vold, J. Magn. Reson. 135 (1998) 541-546.

[42] J.A. Whiles, R.A. Deems, R.R. Vold, E.A. Dennis, Bioorganic Chemistry (2002) in press.

[43] L. Sipos, G. Von Heijne, Eur. Biophys. J. 213 (1993) 1333 1340.

[44] R.A.F. Reithmeier, Curr. Opin. Struct. Biol. 5 (1995) 491-500.

[45] M.J. Espanol, M.H. Saier, Mol. Membr. Biol. 12 (1995) 193 200.

[46] S. Tsang, M.H. Saier, J. Comput. Biol. 3 (1996) 185-190.

[47] B.A. Lewis, D.M. Engelman, J. Mol. Biol. 166 (1983) 211-217.

[48] M. Caffrey, G.W. Feigenson, Biochemistry 20 (1981) 1949-1961.

[49] A. Blume, Biochemistry 2 (1983) 5436-5442. 